International Journal of Advancement in Life Sciences Research

Online ISSN: 2581-4877

journal homepage http://ijalsr.org

Review Article

\title{
Role of nursing professionals in making hospital stay effective and less stressful for patients with ASD: A brief overview
}

\author{
Ishita Mandal ${ }^{1}$, Indrani Basu ${ }^{2}$ and Mitu De ${ }^{3^{*}}$ \\ ${ }^{1}$ Assistant Professor, Department of Nursing, Aliah University, Kolkata, India \\ ${ }^{2}$ Director, Autism Society West Bengal (ASWB), 29/1 Stadium Colony, Mukundapur, Kolkata, India \\ ${ }^{3}$ Associate Professor, Department of Botany, Gurudas College, Kolkata \& Secretary (Hony), Autism Society West \\ Bengal, Kolkata, India
}

Correspondence E-mail :mituaswb@gmail.com *

\begin{abstract}
The hospital may be especially overwhelming to patients with Autism Spectrum Disorder (ASD) and their families. The characteristics and attributes inherently present in patients with ASD provide substantial challenges to nursing care if not recognized, identified and addressed. ASD is a complex disorder and requires preparation, education, and great assessment skills from the nurses for optimal outcomes. Nurses are often the front-line and direct care providers and need to attain the knowledge and skills necessary to understand, interact with and implement excellent care for these individuals. These patients with ASD can present with a range of social and behavioral challenges including difficulty with communication need for structure, and possible aggression and it is difficult to implement care at times. Nowadays the nursing professionals are being increasingly aware of their role in helping to ensure that patients with ASD receive the best possible care. These patients may have difficulty communicating or may find a break in their routine stressful. By understanding ASDrelated challenges and devising ways to make patients as well as their parents or caregivers more comfortable, nurses can make a hospital stay, doctor visit or dental checkup less stressful and more effective. Outcomes improve dramatically when health care professionals obtain the education, expertise, and knowledge necessary to provide such care. In this paper we discuss the common challenges that the nursing staff face while dealing with patients with ASD as well as the difficulties families face. Emphasis will be on the role of nursing professionals in making hospital stay less stressful for individuals with ASD.
\end{abstract}

Keywords: $A S D$, nursing professionals, challenges, hospital stay.

\section{Introduction}

Medical experiences can provoke anxious feelings in children who are typicallydeveloping but individuals with Autism Spectrum Disorder (ASD) face additional challenges, such as difficulty with sensory integration, transitions, flexibility, and communication within their medical experiences (Scarpinato et al, 2010). Though recent research has revealed several aspects of autism, several studies have shown that the care and management of people with ASD remains challenging for doctors, nurse practitioners, registered nurses, and other healthcare providers (Cheak-Zamora \& Teti, 2015; Chiri \& Warfield, 2012). 
ASD is a developmental disorder that affects behavior and communication according to Diagnostic and Statistical Manual of Mental Disorders. 5th ed. (APA, 2013). Autism is considered a "spectrum" disorder because the severity and symptoms vary widely among individuals. Autism Spectrum Disorder (ASD): the enigmatic disorder that knows no boundaries (De, 2014). ASD affects individuals differently. Some individuals diagnosed with ASD are able to independently perform activities of daily living, while others require significant support for basic activities such as communicating and socializing. Healthcare challenges and needs also vary from individual to individual. There is great emphasis on the ideas and efforts of community integration for individuals with ASD in residential, employment, transportation and many other services. However they continue to face significant barriers in accessing quality health care, which has resulted in huge health disparities (Saha, 2019).

The hospital may be especially overwhelming to patients with Autism Spectrum Disorder (ASD) and their families. The characteristics and attributes inherently present in patients with ASD provide substantial challenges to nursing care if not recognized, identified and addressed. Hospitalization for children with ASD may provoke challenging behaviors. Studies have shown that children with ASD have more anxiety and behavioral conduct problems than children without ASD (Gurney et al., 2006). Challenging behaviors are more likely in children with the severe forms of ASD, and include aggression, tantrums, property destruction, hitting, kicking, biting, punching, scratching, and throwing furniture (Hellings et al., 2005; Matson, 2009). These behaviors stress parents, families, nursing personnels and health care providers (Carbone et al., 2010; Scarpinato et al., 2010).

\section{Healthcare for individuals with $A S D$}

Population-based studies of children with ASD report they are also more likely to be hospitalized than typically developing peers, with longer lengths of stays and higher associated costs (Lokhandwala, Khanna, \& WestStrum, 2012). Common comorbidities associated with an ASD diagnosis include gastrointestinal issues, eczema, allergies, asthma, ear and respiratory infections, seizures, and migraines. A large percentage of youth with ASD have medical comorbidities that require frequent visits to the hospital (Kohane et al, 2012)

Irrespective of the current debate about the rise in prevalence of ASD, the significance of this problem is that the increasing rate of ASD (or perhaps, more accurate diagnosing of ASD) foretells that this rising population will potentially contribute significantly to the general population accessing the health care system. Thus, it is imperative for the healthcare provider to be adequately prepared to care for this vulnerable population. several studies have asserted that ASD is associated with extensive comorbidities not limited to intellectual disabilities, sleep disorders, inflammatory bowel disease, developmental disabilities, epilepsy, anxiety, depression, schizophrenia, and other psychiatric disorders (Russell \& McCloskey, 2015; Simonoff et al., 2013; Vohra, Madhavan, Sambamoorthi, \& St Peter, 2014) Given the numerous comorbidities associated with ASD, and the enormous burden on the economy, the significance of this problem must not be ignored. The likelihood of health care providers encountering a pediatric patient with ASD is very high.

Role of Nursing professionals and patients with $A S D$

Studies show that $64.9 \%$ of children with ASD are admitted to the hospital during the first 5 years of life, compared to typically developing children who only have a $48.2 \%$ admission rate (Johnson \& Rodriquez, 2013). The need for medical personnel to have an understanding of ASD and receive training for working with children with ASD continues to become more important. One negative experience during a health care visit for a child with ASD can negatively affect that child's behavior at future visits (Winslow, 2017). Despite this fact, effective training programs in behavior management skills are often unavailable to nurses within the hospital (Drake et al., 2012). Pediatric primary care providers have also often reported that they lack training in managing medical and 
behavioral issues for children with ASD (Bellando, Fussell, \& Lopez, 2016). So the role of nursing professionals in the health care of individuals with ASD is of paramount importance.

Norah L. Johnson, PhD, a Clinical Nurse Specialist in Education Services, Children's Hospital of Wisconsin and an Assistant Professor, Marquette University along with Dana Rodriguez who was a Doctoral Student at Marquette University published systematic review of Literature on the children with ASD in the Pediatric Hospital in 'Pediatric Nursing' in 2013 (Johnson \& Rodriguez 2013). They were of the opinion that 'Hospitalization for any child can be a stressful experience; however, research shows that hospitalization for children with ASD often provokes challenging behaviors that stress parents, families, and health care providers' (Johnson \& Rodriguez, 2013).

Categories of challenging behaviours often encountered by nursing personnels

There are four major categories of challenging behaviors reports in Johnson and Rodriguez' article:

1. non-compliance: This category of noncompliance was extended to include emotional outbursts, temper tantrums, and other behavioral outburst.

2. Hyperactivity: This category of hyperactivity was extended to include impulsive behaviors, elopement, and pestering behaviors.

3. self-stimulatory: Self-stimulatory behaviors also included behaviors revolving around "sensory defensiveness." Sensory defensiveness was defined as "a child's aversion to certain environment elements, such as sounds, textures, and odors" (Johnson \& Rodriguez, 2013).

4. and self-injury.

One of the common reasons, reported by nurses, for children with ASD having tantrums is requesting the child to participate in their health care (Browne, 2006). Overstimulation from people, loud noises, smells, and other factors in the environment are also reported as a common reason for agitation among children with ASD in the hospital. In health care settings, children with ASD may have trouble generalizing skills they use with people they know well to new Health Care Personnels, and they may become anxious and act out (Scarpinato et al., 2010).

Challenging behaviors were also reported because of problems involving communication including: problems communicating physical pain, problems communicating general needs and wants, and problems understanding expectations and directions. Transitions, waiting time and physical discomfort have also been 14 reported as factors that provoke challenging behaviors for children with ASD in a health care setting (Johnson \& Rodriguez, 2013).

Families of children with ASD often experience exhaustion, depression, frustration, and poorphysical health while their child is hospitalized due to the lack of support and understanding among health care professionals (Jolly, 2015). Families have reported feeling that they are not heard by medical professionals. It is important for nurses to have the appropriate training to support both the child with ASD and their families in a health care setting.

\section{Strategies for Dealing with Challenging Behavior}

Parents and Nursing staff recommended many strategies for dealing with challenging behaviors. Many of these strategies reflect are from the nursing staff perspective.

\section{Strategies for non-compliance}

Nurses report that requesting participation of a child with ASD often provokes a tantrum (Thorne, 2007). They have difficulty understanding non-verbal cues, facial expressions, and hand gestures during these en - counters (Scarpinato et al., 2010; Searcy, 2001).

- Communication recommendations to avoid over-stimulation and confusion include using calm, concrete language and avoiding sarcasm or teasing (Debbaudt, 2009). 
- Communication with pictures or a visual schedule is effective as well (Chebuhar, McCarthy, Bosch, \& Baker, 2012).

- Nursing staff need to be patient, reduce the number of commands, and use gentle praise, positive reinforcement (Tucker et al., 2008), and distraction (such as counting or singing) (Galinat, Barcalow, \& Krivda, 2005).

- Other recommendations to decrease stimulation in clude closing doors to limit noise, using "do not disturb signs," clustering nursing interventions, involving parent(s) whenever possible, and spotchecking vital signs instead of continuous monitoring (Inglese, 2009; Scarpinato et al., 2010).

- For procedures, nursing staffs should use a quiet treatment room with durable furniture, hide supplies from view, remove unnecessary equipment, and keep environmental stimuli to a minimum (Souders et al., 2002; Thorne, 2007).

- Other strategies for sensory defensiveness can be personalized to the child by an occupational therapist (Searcy, 2001) and with the input of the parents (Scarpinato et al., 2010).

- An important factor for a tantrum is pain. Determining if a child is in pain may be difficult (Inglese, 2009). Pain can be assessed with the picture pain scales (Golnik \& Maccabee-Ryaboy, 2010) and needs to be treated to prevent outbursts.

- Children with ASD may not cooperate because they tend to like engaging in obsessive activities and routines, and do not want to break them. The rituals give them comfort and should not be interrupted (Browne, 2006; Scarpinato et al., 2010).

\section{Strategies for hyperactivity}

- Planning for physical activity as an outlet for stress and anxiety (Marshall, 2002) and

- Treating the hyperactivity symptoms of ADHD with pharmacology treatments, such as stimulants, which may have side effects and may not be effective (Golnik \& Maccabee-Ryaboy, 2010).
Strategies for sensory defensiveness behaviors

- Nursing staff should not interrupt selfstimulatory behaviors because they serve the purpose of decreasing anxiety (Browne, 2006; Scarpinato et al., 2010).

- Planning ahead by consulting the parent prior to the hospital visit to assess the child's communication; social, sensory, and behavioral skills; successful strategies for compliance, and the child's strengths is recommended (Souders et al., 2002).Knowing what to expect may reduce the child's anxiety (Browne, 2006; Ndubaku, 2018; Scarpinato et al., 2010; Thorne, 2007; Tucker et al., 2008). Preparation includes alerting the child that a change is coming with the use of Now/Then pictures or whatever the parent usually does to signify a change in activity (Chebuhar et al., 2012).

\section{Strategies for self-injury}

- Self injury may be decreased with improved communication methods. Communication strategies include pausing after asking a question to give the child time to process information (Browne, 2006; Debbaudt, 2009). Patience and waiting can be difficult but effective.

Recording of patient profile by nursing professionals

Adriane A. Jolly in her article in the 2015 JanFeb issue of Pediatric Nursing formulated a 'Template for Admission Assessment Tool for the Child with Autism Spectrum Disorder.' It is given in Table 1. Communication gets lost among providers during shift change. Inef fective handoffs can place all patients at risk, not just the child with ASD (Friesen, White, \& Byers, 2008). Incomplete handoffs can be particularly detrimental to the safety of the child with ASD and the personnel caring for the child if critical information on necessary methods of communication, the child's triggers that may lead to increasing agitation and anxiety, the daily schedule, and the child's preferences are not clearly or completely relayed. The nurse admitting the child with ASD to the inpatient unit should record a 
patient profile to decrease the chance that the information is lost during shift change (Inglese, 2009). Nursing professionals should allow parents to bring in clothes, belongings, and food from home to make the child more comfortable (Jolly, 2015).

Table 1. Template for Admission Assessment Tool for the Child with Autism Spectrum Disorder (Jolly, 2015)

\begin{tabular}{|l|l|}
\hline $\begin{array}{l}\text { Sl. } \\
\text { No. }\end{array}$ & Questions that Nursing Professionals should ask parents during admitting the patient with ASD \\
\hline 1 & What causes your child to have increased anxiety? Please list common triggers. \\
\hline 2 & $\begin{array}{l}\text { What is the best method to communicate with your child? a. Verbal, Picture, Sign Language, } \\
\text { Other } \\
\text { b. Are there any special communication tools (ex: spell board) that we can obtain during your } \\
\text { child's stay? }\end{array}$ \\
\hline 3 & How should staff members approach your child? \\
\hline 4 & Is your child particularly sensitive to touch, sound, smell, sight, or taste? \\
\hline 5 & Does your child have any obsessive/restrictive behaviors? \\
\hline 6 & How does your child demonstrate if he or she is in pain? \\
\hline 7 & What are your child's early signs of increasing frustration and anxiety? \\
\hline 8 & What are your best methods to comfort and deescalate your child? \\
\hline 9 & What are your child's strengths? \\
\hline 10 & What is your child's home routine? \\
\hline
\end{tabular}

Jayne Jennings Dunlap who is a family nurse practitioner at Baylor Scottand White- Belton South Family Medicine Clinic in Belton, Texas, and a doctor of nursing practice student at Texas Christian University in Fort Worth stated that 'Successful care depends on communication with patients, families and caregivers.'

In an article (Dunlap, 2018) a long list of practice tips arranged category wise for the nursing staff was listed which is given below.

Dunlap's List: The nursing staffs are advised to keep this advice in mind when caring for a patient with autism spectrum disorder (ASD).

\section{Appointment guidance}

Although special arrangements may not be possible in emergent situations, strive to:

- arrange an informal trip for the patient and family or caregiver to the healthcare setting before the first appointment

- take and share pictures of key facility areas, equipment, and healthcare staff

- allow individuals with ASD to watch a family member undergo similar exams first
- ask about healthcare history and previous healthcare experiences

- provide extra time to accommodate any additional patient needs, if possible

- share strategies with staff to best communicate and interact with patients with ASD

- schedule the patient as the first or last appointment of the day when possible.

\section{Interaction tips}

- Explain what you will do before starting any examination or procedure.

- Show the equipment to patients and provide them with an opportunity to visually explore and touch it.

- Provide a picture illustrating what will happen.

- Allow caregivers to stay in the room as appropriate.

\section{Communication tips}

- Speak in short sentences with clear, precise language.

- Use concrete, literal words. 
- Set up a plan (First, then

- Ask patients and caregivers for specific information, and avoid abstract questions.

- Give exact instructions directly (for example, instead of asking "Can you stand up?" say "Stand up please.")

- Avoid figures of speech, especially metaphors, idioms, irony, or words with double meaning.

- Be aware that body language and facial expressions may not be understood.

- Praise specific behaviors rather than providing general praise.

- Assess patient understanding. You can ask the patient to repeat back information, ask it again, or use a fill-in-the blank statement as appropriate, which may allow the patient to answer questions and discuss or indicate items or experiences (for example, "A part of your body that hurts is ").

\section{Sensory tips}

- Meet with patients in a quiet room whenever possible.

- Use natural light or dim fluorescent lighting, if possible.

- Talk to one person at a time.

- Tell patients that you will touch them before you do so.

- Avoid unnecessary touching.

- Encourage patients and caregivers to bring objects to regulate sensory stimuli (for example, headphones, sunglasses, or sensory toys).

- Expect atypical pain thresholds (decreased or heightened).

\section{Patient response}

- Don't expect eye contact, especially in distressing situations.

- Allow patients extra time for processing information.

- Recognize that patients with ASD may not be able to understand others' perspectives but may expect you to understand theirs.
- Stimming (pacing, hand flapping, fidgeting, or rocking) may be present and is a coping mechanism.

- Expect that patients may invade others' personal space or require increased personal space for themselves.

By understanding ASD-related challenges and devising ways to make patients as well as their parents or caregivers more comfortable, nurses can make a hospital stay, doctor visit or dental check up less stressful and more effective. Outcomes improve dramatically when health care professionals obtain the education, expertise, and knowledge necessary to provide such care. Incorporating these strategies into the care of the hospitalized child with ASD may contribute to a safer, less stressful and more pleasant hospital admission for the child, the family, and hospital personnel.

\section{Discussion}

As the prevalence of children diagnosed with Autism Spectrum Disorder (ASD) continues to rise, the importance of providing nursing professionals in health care and educational settings with training for working with individuals with ASD also continues to rise (Winslow, 2017). Successfully caring for the child with ASD requires the nurse to acknowledge that this patient population is unique and requires a thorough assessment, multidisciplinary planning, advanced knowledge of the child's special needs, and close collaboration with the child's family (Jolly, 2015). The pediatric nurse is often unaware of or unprepared to offer the care that this special population requires. It is recommended that nursing professionals need to understand the needs of the patients with ASD during their training days. To test the effectiveness of interventions, HCPs need a valid and reliable standardized behavior observation tool to accurately measure children's behavioral outcomes. The development of such a tool is difficult given all the various conditions included in the autism spectrum (Johnson \& Rodriquez, 2013). 
The pediatric nurse should advocate for the patient and involve the multidisciplinary team from the beginning of the admission of the child with ASD. The nurse should encourage a family meeting outside of the patient's room where physical, occupational, and speech therapists can learn the child's level of activity and assist with the creation of the schedule and plan. Nutritionists should be involved to ensure that the child is presented with foods he or she will likely eat (Jolly, 2015).

In the care of children with ASD the Health Care Professionals need to consider the manifestations of ASD in the child and how to accommodate for each child. Taking the wide variation of functioning between children with ASD into consideration it is necessary to see each child as an individual and care for each child according to person-centred care (Cronenwett, 2009). Care without knowledge and without consideration to the needs of the specific child can cause great suffering in both the child and the parents (Lindberg and Eriksson, 2012).

ASD is a complex disorder and requires preparation, education, and great assessment skills from the nurses for optimal outcomes. Nurses are often the front-line and direct care providers and need to attain the knowledge

\section{References}

American Psychiatric Association. 2013. Diagnostic and Statistical Manual of Mental Disorders. 5th ed. Washington, DC: American Psychiatric Association.

Bellando, J., Fussell, J. J., \& Lopez, M. 2016. Autism speaks toolkits. Clinical Pediatrics, 55(2), 171-175 pp.

Browne, M. E. 2006. Communicating with the child who has autistic spectrum disorder: A practical introduction. (Cover story). Pediatric Nursing, 18(1), 14-17 pp.

Carbone, P., Farley, M., \& Davis, T.E. 2010. Primary care for children with autism. American Family Physician, 81(4), 461 pp.

Cheak-Zamora, N. C., \& Teti, M. 2015. "You think it's hard now ... It gets much harder for our children": Youth with autism and their and skills necessary to understand, interact with and implement excellent care for these individuals. A well informed trained nursing professional with prior knowledge about ASD and strategies for combating challenging behaviours may make a great difference to a patient with ASD. Nowadays the nursing professionals are being increasingly aware of their role in helping to ensure that patients with ASD receive the best possible care. From recent research and literature review it is clear that the role of nursing professionals in making hospital stay effective and less stressful for patients with ASD is a vital one.

\section{Conflicts of Interest}

The authors declare that there are no conflicts of interest regarding the publication of this work.

\section{Acknowledgement:}

The authors wish to thank some parents who take intervention at Autism Society West Bengal, Kolkata, India for sharing their experience of hospital stay for their child. The authors would also like to thank some nursing professionals at the Department of Nursing, Aliah University, Kolkata, India for sharing their personal experiences while dealing with patients with ASD.

caregiver's perspectives of health care transition services. Sage, 19(8), 992-1001 pp.

Chebuhar, A., McCarthy, A.M., Bosch, J., \& Baker, S. 2012. Using picture schedules in medical settings for patients with an autism spectrum disorder. Journal of Pediatric Nursing; Apr;28(2):125-34 pp.

Chiri, G., \& Warfield, M. E. 2012. Unmet need and problems accessing core health care services for children with autism spectrum disorder. Maternal Child Health Journal, 16(5), 1081-1091 pp.

Cronenwett, L., et al. 2009. Quality and safety education for advanced nursing practice. Nursing Outlook, 57(6): p. 338-348

De, M. 2014. Autism Spectrum Disorder (ASD): the enigmatic disorder that knows no boundaries. The Beats of Natural Sciences. 
(ISSN-2348-7615) Vol. 1, Issue 3 (September), Article No. 4. pp 1-15.

Debbaudt, D. 2009. Patients with autism and other high risks: A growing challenge for healthcare security. Journal of Healthcare Protection Management, 25(1), 14-26 pp.

Drake, J., Johnson, N., Stoneck, A. V., Martinez, D. M., \& Massey, M. 2012. Evaluation of a coping kit for children with challenging behaviors in a pediatric hospital. Pediatric Nursing, 38(4), 215-221 pp.

Dunlap, Jayne Jennings, 2018. Interacting with individuals on the Autism spectrum. American Nurse Today. Vol. 13. No. 4.April issue pp 1619.

Galinat, K., Barcalow, K., \& Krivda, B. 2005. Caring for children with autism in the school setting. The Journal of School Nursing, 21(4), 208-217 pp.

Golnik, A., \& Maccabee-Ryaboy, N. 2010. Autism: Clinical pearls for primary care. Contemporary Pediatrics, November, 42-59 pp.

Gurney, J., McPheeters, M., \& Davis, M. 2006. Parental report of health conditions and health care use among children with and without autism. Archives of Pediatric and Adolescent Medicine, 160, 825-830 pp.

Hellings, J., Nickel, E., Weckbaugh, M., McCarter, K., Mosier, M., \& Schroeder, S. 2005. The overt aggression scale in outpatient youth with autistic disorder: Preliminary findings. Journal of Neuro - psychiatry Clinical Neuroscience, 17(1), 29-35 pp.

Inglese, M.D. 2009. Caring for children with autism spectrum disorder. Part II: Screening, diagnosis, and management. Journal of Pediatric Nursing, 24(1), 49-59 pp.

Johnson, N. L., \& Rodriguez, D. 2013. Children with autism spectrum disorder at a pediatric hospital: A systematic review of the literature. Pediatric Nursing, 39(3), 131-141 pp.

Jolly, A. A. 2015. Handle with care: Top ten tips a nurse should know before caring for a hospitalized child with autism spectrum disorder. Pediatric Nursing, 41(1), 11-22 pp.
Kohane IS, McMurry A, Weber G, et al. 2012. The co-morbidity burden of children and young adults with autism spectrum disorders. PLoS ONE.; 7:e33224.

Lindberg, S., I. von Post, and K. Eriksson. 2012. The experiences of parents of children with severe autism in connection with their children's anaesthetics, in the presence and absence of the perioperative dialogue: a hermeneutic study. Scandinavian Journal of Caring Sciences, 26(4): p. 627-634 pp.

Lokhandwala, T., Khanna, R., \& West-Strum, D. 2012. Hospitalization burden among individuals with Autism. Journal of Autism and Developmental Disor - ders, 42(1), 95-104 pp.

Matson, J.L. 2009. Aggression and tantrums in children with autism: A review of behavioral treatments and maintaining variables. Journal of Mental Health Research in Intellectual Disabilities, 2, 169-187 pp.

Marshall, M. 2002. Asperger's syndrome: Implications for nursing practice. Issues in Mental Health Nursing, 23, 605-615 pp.

Ndubaku, Ugo. 2018. "Barriers to Providing Quality Care for Pediatric Patients with Autism Spectrum Disorder as Identified byBaccalaureate Prepared Registered Nurses: A Basis to Enhance Professional Practice." Doctoral Projects. 74.DOI: https://doi.org/10.31979/etd.9ck7-

c9drhttps://scholarworks.sjsu.edu/etd_doctoral 174

Russell, S., \& McCloskey, C. R. 2015. Parents perceptions of care received by children with an autism spectrum disorder. Journal of Pediatric Nursing, 31, 21-31.

Saha, Animita C. 2019. Our lives, our healthcare: How autism can affect healthcare. In, Understanding Autism: Through the Lens of parents and Professionals. ISBN 978-9381574-92-8. Edited by Subir Kumar Dutta and Mitu De published by The Asiatic Society, 1 Park Street, Kolkata. 158-167 pp.

Scarpinato, N., Bradley, J., Kurbjun, K., Bateman, X., Holtzer, B., \& Ely, B. 2010. Caring for the child with an autism spectrum disorder in the acute care setting. Journal for Specialists in Pediatric Nursing, 15(3), 244$254 \mathrm{pp}$. 
Searcy, E. 2001. Helping the patient who has pervasive developmental disorder. Journal of the American Academy of Physician Assistants, 14(10), 39 pp.

Simonoff, E., Jones, C. R. G., Baird, G., Pickles, A., Happe, F., \& Charman, T. 2013. The persistence and stability of psychiatric problems in adolescents with Autism Spectrum Disorders. Journal of Child Psychology and Psychiatry, 54(2), 186-194 pp.

Souders, M., Freeman, K., DePaul, D., \& Levy, S.E. 2002. Caring for children and adolescents with autism who require challenging procedures. Pediatric Nursing, 28(6), 555-562 pp.

Thorne, A. 2007. Are you ready to give care to a child with autism? Nursing, 37, 59- 61 pp.
Tucker, S., Derscheid, D., Odegarden, S., \& Olson, M. 2008. Evidence-based training for enhancing psychiatric nurses' child behavior management skills. Journal for Nurses in Staff Development, 24(2), 75-85 pp.

Vohra, R., Madhavan, S., Sambamoorthi, U., \& St Peter, C. 2014. Access to services, quality of care, and family impact for children with autism, other developmental disabilities, and other mental health conditions. Autism, 18(7), 815-826 pp.

Winslow, Paige M., "Preparation of Nursing Students to Address Challenging Behaviors of Patients with Autism Spectrum Disorder in Health Care Settings" 2017. Project for Master of Science at St. Cloud State University as Culminating Projects in Child and Family Studies pp 46. 